OPEN ACCESS

Edited by:

Jianguang $\mathrm{Ji}$

Lund University, Sweden

Reviewed by:

Zhijun Dai,

Zhejiang University, China

Shun He,

Cancer Hospital (CAMS), China

*Correspondence:

Haifeng Hou

hfhou@163.com

Youxin Wang

wangy@ccmu.edu.cn

tThese authors have contributed equally to this work

Specialty section:

This article was submitted to Cancer Epidemiology and Prevention, a section of the journal

Frontiers in Oncology

Received: 23 February 2019 Accepted: 05 June 2019

Published: 27 June 2019

Citation:

Hou H, Meng Z, Zhao X, Ding G, Sun $M$, Wang W and Wang $Y$ (2019)

Survival of Esophageal Cancer in

China: A Pooled Analysis on Hospital-Based Studies From 2000 to

2018. Front. Oncol. 9:548.

doi: 10.3389/fonc.2019.00548

\section{Survival of Esophageal Cancer in China: A Pooled Analysis on Hospital-Based Studies From 2000 to 2018}

\author{
Haifeng Hou ${ }^{1 * t}$, Zixiu Meng ${ }^{1 \dagger}$, Xuan Zhao ${ }^{1 \dagger}$, Guoyong Ding ${ }^{1}$, Ming Sun ${ }^{2}$, Wei Wang ${ }^{1,3}$ and \\ Youxin Wang ${ }^{2 \star}$ \\ ${ }^{1}$ School of Public Health, Taishan Medical University, Taian, China, ${ }^{2}$ Beijing Key Laboratory of Clinical Epidemiology, School \\ of Public Health, Capital Medical University, Beijing, China, ${ }^{3}$ School of Medical and Health Sciences, Edith Cowan University, \\ Perth, WA, Australia
}

Background: Esophageal cancer (EC) causes more than 400 thousand deaths per year, and half of them occur in China. There are discrepancies regarding the survival of EC patients between population-based surveillance studies and hospital-based studies.

Objectives: We aimed to synthesize the survival data from hospital-based EC studies in the Chinese population from 2000 to 2018 and to compare the survival rates between EC patients with different clinical classifications.

Methods: The protocol of this systematic review was registered in PROSPERO (CRD-42019121559). We searched Embase, PubMed, CNKI, and Wanfang databases for studies published between January 1, 2000 and December 31, 2018. We calculated the pooled survival rates and 95\% confidence intervals (Cls) by Stata software (V14.0).

Results: Our literature search identified 933 studies, of which 331 studies with 79,777 EC patients met the inclusion criteria and were included in meta-analyses. The pooled survival rates were $74.1 \%$ (95\% Cl: 72.6-75.7\%) for 1-year survival, 49.0\% (95\% Cl: 44.2-53.8\%) for 2-years survival, 46.0\% (95\% Cl: 42.6-49.5\%) for 3-years survival, and $40.1 \%$ (95\% Cl: 33.7-46.4\%) for 5-years survival. An increased tendency toward EC survival was verified from 2000 to 2018. In addition, discrepancies were observed between EC patients with different clinical classifications (e.g., stages, histologic types, and cancer sites).

Conclusions: Our findings showed a higher survival rate in hospital-based studies than population-based surveillance studies. Although this hospital-based study is subject to potential representability and publication bias, it offers insight into the prognosis of patients with EC in China.

Keywords: esophageal cancer, survival, meta-analysis, hospital-based study, China 


\section{INTRODUCTION}

Esophageal cancer (EC) is among the top causes of cancerrelated mortality globally, resulting in more than 400 thousand deaths each year (1). Because its incidence is increasing, EC remains a global concern, especially in under-developed regions (2). Annually, more than half of new cases are diagnosed in China, where a so-called EC belt permanently exists around Taihang Mountain $(3,4)$. EC ranks fifth in incidence $(21.17$ per $100,000)$, and fourth in mortality (15.58 per 100,000) among all malignant tumors in China $(5,6)$. Carcinogen exposure (e.g., nitrosamines and their precursors, fungi, trace minerals, and polycyclic hydrocarbons), nutritional deficiency, lifestyles, and genetics contribute to the multistage development of EC through (1) localized injury, (2) inflammation, (3) mutagenesis, and (4) carcinogenesis $(7,8)$.

Although strategies for preventing EC are necessary, measures to reduce morbidity or improve survival are also extremely important. Therefore, a national screening program was launched in 2005 in areas in China with a high prevalence of EC, especially rural regions (9). This program includes endoscopic examination for high-risk individuals and early surgery in the treatment of patients with EC (5). Several studies have reported a 50\% decrease in EC deaths among subjects served in this healthcare program $(9,10)$. Also, a nationwide population-based cancer registry system was initiated to collect cancer data across China in $2002(11,12)$. These population-based survival databases enable both temporal and spatial surveillance of the overall state of different cancers (13). However, incomplete follow-up for registered individuals can bias survival estimates in population-based surveillance studies. When the registered data do not cover all eligible cancer deaths, the survival statistics might be overestimated, particularly for cancers with high fatality rates (14). Meanwhile, many clinical investigations have reported the prognosis of patients with cancer within hospitals according to clinical research criteria. These hospital-based survival investigations can reflect comprehensive treatment effectiveness with relatively lower loss to follow-up than population-based studies (15). Discrepancies exist regarding the survival of EC patients between reports from the national cancer registry system and hospital-based studies, as well as between hospital-based studies. The present study aimed to systematically synthesize all eligible survival data reported in hospital-based clinical studies in the Chinese population in order to estimate the prognosis of hospitalized patients with EC.

\section{MATERIALS AND METHODS}

The systematic review and meta-analyses were conducted in accordance with the criteria of the Preferred Reporting Items for Systematic Reviews and Meta-Analyses (PRISMA) (16). The reported PRISMA Checklist is available in the online Table S1. As a systematic review, the protocol was registered in PROSPERO (No. CRD-42019121559, https://www.crd.york. ac.uk/PROSPERO/).

\section{Literature Search Strategy}

We searched Embase, PubMed, and Web of Science for English language literature, as well as CNKI and Wanfang for Chinese literature published between January 1, 2000 and December 31, 2018. The combination of "China," "Chinese," "survival," "hospital," "esophageal cancer," "carcinoma of esophagus," "esophageal carcinoma," "esophagus cancer," "cancer of esophagus," “esophageal cancer," “carcinoma of esophagus," "esophageal carcinoma," "esophagus cancer," and "cancer of esophagus" in English or Chinese were used in the literature search. References cited in the included articles were further reviewed. The detailed search strategy is shown in Table S2.

\section{Selection Criteria and Quality Assessment}

First, two authors (XZ and ZM) independently reviewed the titles and abstracts of the retrieved publications. Second, the full text and online supplementary data were read to determine the eligibility and quality of the literature (Table S3). Any uncertainties and discrepancies were resolved by the third investigator $(\mathrm{HH})$ through discussion. The inclusion criteria were (1) EC was diagnosed by pathological examination; (2) the following data were available: number of included EC patients, number of survived cases, or survival rates of EC patients; (3) the survival data were obtained from hospital-based studies that assessed the prognosis of hospitalized inpatients; (4) the ethnicity of all EC patients was Chinese. For studies reporting on both Chinese and other populations, only the data for the Chinese population were included. The exclusion criteria were as follows: (1) in vitro studies or animal studies; (2) reviews; (3) studies that did not refer to a Chinese population; (4) studies without survival data; (5) studies on community-based populations; (6) low-quality studies.

\section{Data Extraction}

The following data were extracted from included studies: (1) first author's name, publication year, characteristics of EC patients (e.g., age, gender, region, cancer site, clinical stage, and clinical type), survival rate or number of survival cases, and study design. If data of a specific population were reported in several studies or published more than once, the most recently published or the largest sampled study was included. Data in each subgroup (study design, gender, regions, cancer site, clinical type, and stage) were also extracted for subgroup analyses.

\section{Statistical Analysis}

The pooled survival rate and $95 \%$ confidence interval (CI) of EC were calculated by Stata V14.0 (Stata Corp, College Station, TX, USA). Cochran's Q-test for heterogeneity, complemented by the $I^{2}$ statistic, was implemented to measure the level of heterogeneity across original studies. A $P>0.10$ and $I^{2}<50 \%$ indicated no significant heterogeneity, and a fixed effect model was utilized to calculate the pooled survival rate. Otherwise, significant heterogeneity was assumed, then the random effect model was carried out subsequently by the DerSimonianLaird method (17). Subgroup analyses on gender, cancer site, clinical type, and stage, temporal trend, and study design were conducted, respectively. To assess the strength and stability 


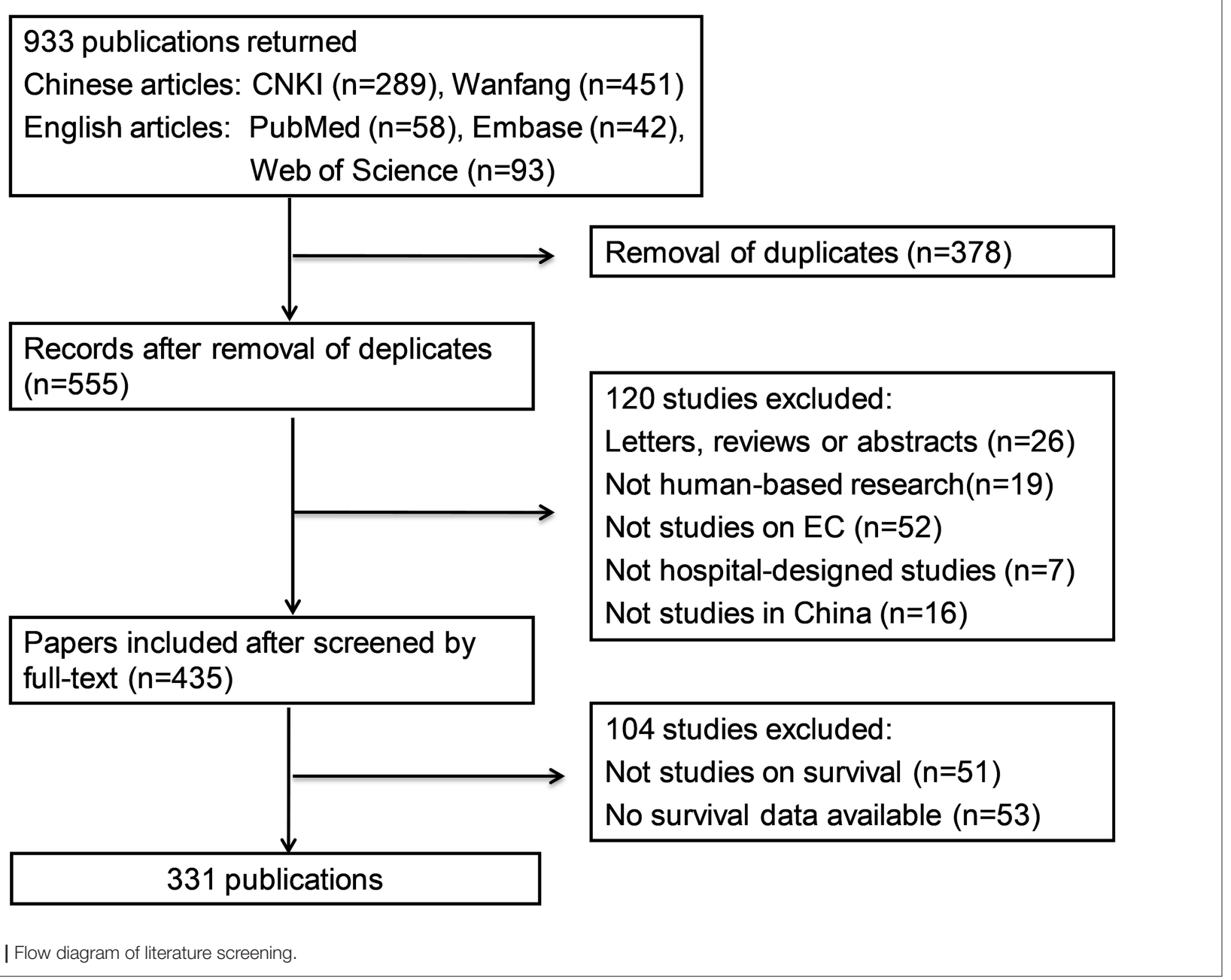

of pooled results, sensitivity analyses were also conducted by successive removal of an eligible study each time. The publication bias was estimated by funnel plot asymmetry and Begg's adjusted rank correlation test. A $P$-value $<0.05$ was considered to represent statistical significance.

\section{RESULTS}

\section{Systematic Review and Eligible Studies}

Our literature search identified 933 publications, of which 289 were from CNKI, 451 were from Wanfang, 42 were from Embase, 58 were from PubMed, and 93 were from Web of Science. Two authors (ZM and ZX) reviewed the retrieved literature independently and then excluded 378 reduplicate publications. Among the 555 publications involved in the abstract and fulltext review, 26 were not original studies, 19 were not humanbased research, 52 were not in reference to EC, seven were not designed as hospital-based studies, 16 were not conducted among a Chinese population, 51 were not investigations on survival or prognosis, and 53 did not provide appropriate survival data. Finally, 331 publications with 79,777 EC patients $(52,273$ males/21,874 females/5,630 unavailable; mean age 51.8 years) that met the predefined criteria were included in our study. The flow diagram for screening articles is shown in Figure $\mathbf{1}$. The distributions of the studies included are shown in Figure 2 and additional details are provided in Table S4. There were 104 prospective studies and 227 retrospective studies. A total of 118 studies reported survival of patients with esophageal squamous cell carcinoma (ESCC), eight reported survival of patients with esophageal small cell carcinoma (SCC), two reported survival of patients with esophageal adenocarcinoma (EAC), 119 reported the combined data of more than one histologic type, and 84 studies did not mention the histologic classifications. Of the 331 studies, 210 were conducted in the high prevalence areas of China (e.g., Fujian, Guangdong, Henan, Jiangsu, Shandong, Shanxi, Shaanxi, and Sichuan provinces).

\section{Pooled Results of EC Survival}

As shown in Table 1, the observed survival rates in all eligible studies were synthesized by a random effect model. The overall pooled results were $74.1 \%$ (95\% CI: $72.6-75.7 \%$ ) for 1 -year survival, $49.0 \%$ (95\% CI: $44.2-53.8 \%$ ) for 2-years survival, $46.0 \%$ (95\% CI: 42.6-49.5\%) for 3-years survival, and 40.1\% (95\% CI: $33.7-46.4 \%$ ) for 5-years survival. 


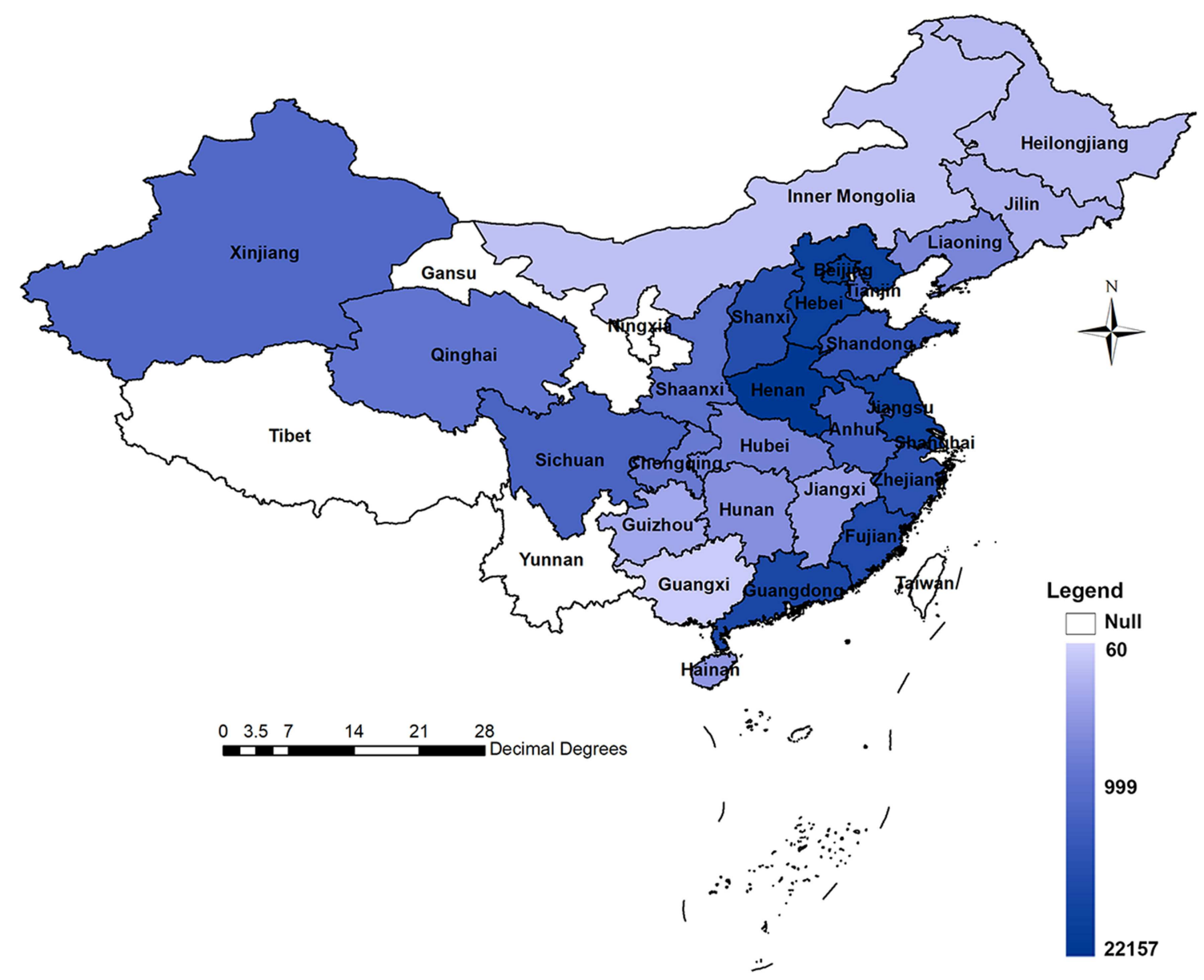

FIGURE 2 | Distribution of included studies.

TABLE 1 | Pooled survival and 95\% $\mathrm{Cl}$ of esophageal cancer in low and high prevalence areas.

\begin{tabular}{|c|c|c|c|c|c|c|}
\hline Survival & \multicolumn{2}{|c|}{ Low prevalence areas } & \multicolumn{2}{|c|}{ High prevalence areas } & \multicolumn{2}{|c|}{ Overall } \\
\hline 2 -years & 62 & $47.6(40.2-55.0)^{\mathrm{a}}$ & 63 & $50.4(45.0-55.8)^{\mathrm{a}}$ & 125 & $49.0(44.2-53.8)^{\mathrm{a}}$ \\
\hline 3-years & 107 & $45.8(40.2-51.5)^{\mathrm{a}}$ & 104 & $46.2(42.5-49.9)^{\mathrm{a}}$ & 211 & $46.0(42.6-49.5)^{\mathrm{a}}$ \\
\hline 5 -years & 47 & $41.3(33.0-49.6)^{a}$ & 26 & $37.1(29.3-45.0)^{\mathrm{ab}}$ & 73 & $40.1(33.7-46.4)^{a}$ \\
\hline
\end{tabular}

$S R$, survival rate; $C l$, confidence interval; $N$, number of included studies; Between-group comparisons: ${ }^{a} P<0.05$ compared with 1 -year survival; ${ }^{b} P<0.05$ compared with 2-years survival.

To examine the survival profiles from 2000 to 2018, we conducted analyses on time-trend survival of patients with EC. As shown in Figure 3 and Table S5, a tendency for increased survival was evidenced by the pooled survival rates from 2006 to 2018. The same trends were found in both men and women (Tables S6, S7), except that the 5-years survival rates among men decreased from 2006 to 2015.

Subgroup analysis on study design (Figure 4 and Table S8) indicated that the pooled survival rates in retrospective studies were $75.3 \%$ (95\% CI: 73.5-77.2\%) for 1-year survival, 49.6\% (95\% CI: $43.3-55.9 \%)$ for 2 -years survival, $45.5 \%$ (95\% CI: $41.4-49.6 \%)$ for 3-years survival, and 39.8\% (95\% CI: 33.0-46.7\%) for 5-years survival. Meanwhile, the pooled survival rates in prospective studies were $71.9 \%$ (95\% CI: 68.6-75.2\%) for 1-year survival, $48.1 \%$ (95\% CI: $41.5-54.8 \%$ ) for 2-years survival, $47.6 \%$ (95\% CI: $42.8-52.3 \%$ ) for 3 -years survival, and $42.6 \%$ (95\% CI: $34.7-$ $50.4 \%$ ) for 5 -years survival. Moreover, subgroup analyses by sex showed higher rates of 2-, 3-, and 5-years survival among women 


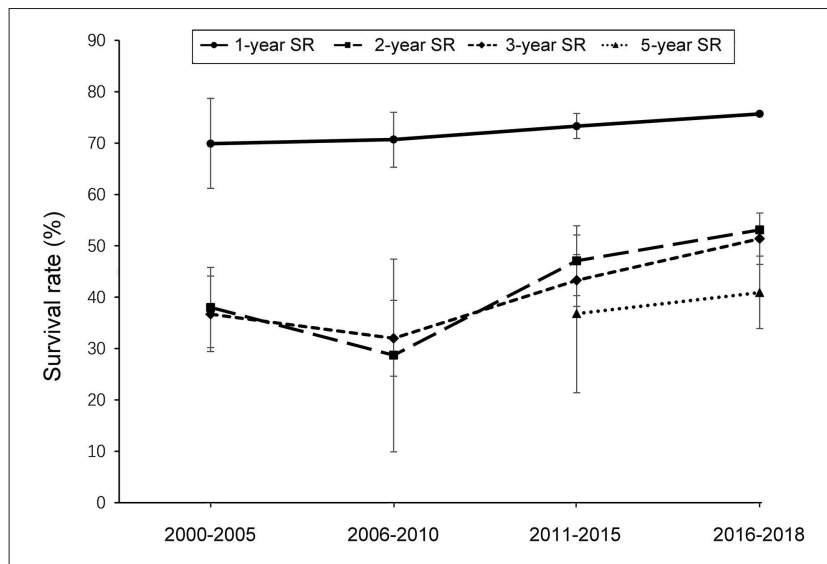

FIGURE 3 | Temporal trends of survival of esophageal cancer from 2000 to 2018. Between-group comparisons: the 1-year survival rates are significantly higher than 2-, 3-, and 5-years survival rates between 2000 and $2018(P<0.05)$.

Retrospective studies
One-year SR
Two-year SR
Three-year SR
Four-year SR

than for those of men, while the differences were not statistically significant (Tables S6, S7).

We also synthesized survival rates in the high prevalence areas of China (e.g., Fujian, Guangdong, Henan, Jiangsu, Shandong, Shanxi, Shaanxi, and Sichuan provinces), where the incidence rates of EC are higher than other provinces. The pooled survival rates in these high prevalence areas were $74.2 \%$ (95\% CI: $71.8-$ $76.5 \%$ ) for 1-year survival, $50.4 \%$ (95\% CI: 45-55.8\%) for 2-years survival, $46.2 \%$ (95\% CI: 42.5-49.9\%) for 3-years survival, and $37.1 \%$ (95\% CI: $29.3-45.0 \%$ ) for 5-years survival (Table 1). The prognosis of EC in high prevalence areas was similar with low prevalence areas.

\section{Subgroup Analyses of EC Survival by Clinical Classifications}

Thirty-five studies that involved 13,055 patients reported the TNM stage of ED. As shown in Table 2, the pooled analyses
TABLE 2 | Pooled survival and 95\% Cl of esophageal cancer in different clinical stages.

\begin{tabular}{|c|c|c|c|}
\hline Categories & Stage & $N$ & SR (\%) \\
\hline \multirow[t]{5}{*}{ 1-year survival } & T1 & 6 & $95.6(93.5-97.7)$ \\
\hline & T2 & 8 & $84.7(79.3-90.0)^{\mathrm{a}}$ \\
\hline & T3 & 12 & $74.6(68.8-80.4)^{\mathrm{a}}$ \\
\hline & T4 & 17 & $52.8(39.9-65.7)^{\mathrm{abc}}$ \\
\hline & Overall & 43 & $71.7(66.5-76.9)$ \\
\hline \multirow[t]{5}{*}{ 2-years survival } & $\mathrm{T} 1$ & 3 & 87.0 (71.0-103.0) \\
\hline & T2 & 3 & $51.4(39.9-62.9)^{\mathrm{a}}$ \\
\hline & T3 & 4 & $34.5(28.8-40.2)^{\mathrm{a}}$ \\
\hline & $\mathrm{T} 4$ & 2 & $11.4(2.2-25.1)^{\mathrm{abc}}$ \\
\hline & Overall & 12 & $41.8(13.7-69.8)$ \\
\hline \multirow[t]{5}{*}{ 3-years survival } & $\mathrm{T} 1$ & 10 & 81.0 (75.5-86.4) \\
\hline & $\mathrm{T} 2$ & 13 & $61.5(53.6-69.3)^{\mathrm{a}}$ \\
\hline & T3 & 17 & $45.0(36.9-53.1)^{a b}$ \\
\hline & $\mathrm{T} 4$ & 11 & $36.3(21.8-50.7)^{\mathrm{ab}}$ \\
\hline & Overall & 51 & $53.3(46.5-60.1)$ \\
\hline \multirow[t]{5}{*}{ 5-years survival } & T1 & 13 & 75.2 (68.4-82.0) \\
\hline & $\mathrm{T} 2$ & 14 & $51.6(43.3-59.9)^{a}$ \\
\hline & T3 & 16 & $38.5(30.6-46.4)^{a}$ \\
\hline & $\mathrm{T} 4$ & 10 & $25.4(14.9-36.0)^{a b}$ \\
\hline & Overall & 53 & $48.4(41.6-55.1)$ \\
\hline
\end{tabular}

SR, survival rate; $\mathrm{Cl}$, confidence interval; $N$, number of included studies. Between-group comparisons: ${ }^{a} P<0.05$ compared with $T 1$ group; ${ }^{b} P<0.05$ compared with T2 group; ${ }^{c} P<0.05$ compared with T3 group.

showed a decreasing linear trend of survival (including 1-, 2-, 3-, and 5-years survival) for patients with T1, T2, T3, and T4 stage EC. A decreased trend was found in accordance with the TNM stage classifications. The 5-years survival of patients with stage T4 EC was $25.4 \%$ (95\% CI: $14.9-36.0 \%$ ), which was significantly lower than the survival for patients with stage T1 EC (75.2\%, 95\% CI: 68.4-82.0\%).

The major histologic type of EC in China is ESCC, which accounts for $90 \%$ of new cases, while adenocarcinoma is more common in Western countries (18-20). The pooled results (Table 3) show that the 5-years survival rate of SCC (15.5\%, 95\% CI: 12.4-18.6\%) was significantly lower than that of ESCC (41.7\%, 95\% CI: 32.4-51.0\%). The 1- and 3-years survival rates of SCC were significantly lower as well. However, only two studies reported survival of EAC, for which the pooled 1- and 3-years survival rates were $80.9 \%$ (95\% CI: 62.3-99.5\%) and $45.7(95 \%$ CI: $23.2-68.2 \%)$, respectively.

We performed a subgroup meta-analysis on four sites of the original tumors. As shown in Table 4, 1-, 3-, and 5-years survival rates were lower among patients with hypopharynx and cervical EC than patients with thoracic EC.

\section{Results of Publication Bias and Sensitivity Analyses}

As shown in Figure 5, the funnel plot analyses and Begg's tests indicated no publication bias in the meta-analyses of 3- and 5years survival. However, significant publication bias was detected in both 1- and 2-years survival analyses. 
TABLE 3 | Pooled survival and 95\% Cl of esophageal cancer of different histologic types.

\begin{tabular}{|c|c|c|c|}
\hline Categories & Types & $N$ & SR (\%) \\
\hline \multirow[t]{5}{*}{ 1-year survival } & Combined & 145 & $74.1(72.1-76.0)$ \\
\hline & ESCC & 80 & 75.3 (72.7-78.0) \\
\hline & SCC & 5 & $55.6(40.4-70.8)^{\mathrm{a}}$ \\
\hline & EAC & 2 & $80.9(62.3-99.5)$ \\
\hline & Overall & 232 & $74.1(72.6-75.7)$ \\
\hline \multirow[t]{5}{*}{ 2-years survival } & Combined & 79 & $50.40(44.5-56.2)$ \\
\hline & ESCC & 41 & $48.70(39.9-57.6)$ \\
\hline & SCC & 4 & $24.40(4.6-44.2)$ \\
\hline & EAC & 1 & - \\
\hline & Overall & 125 & $49.00(44.2-53.8)$ \\
\hline \multirow[t]{5}{*}{ 3-years survival } & Combined & 127 & $47.8(43.2-52.3)$ \\
\hline & ESCC & 78 & $44.6(39.4-49.7)$ \\
\hline & SCC & 4 & $18.8(2.6-35.0)^{\mathrm{a}}$ \\
\hline & EAC & 2 & $45.7(23.2-68.2)$ \\
\hline & Overall & 210 & $46.0(42.6-49.5)$ \\
\hline \multirow[t]{5}{*}{ 5-years survival } & Combined & 37 & $41.30(31.7-50.9)$ \\
\hline & ESCC & 31 & $41.70(32.4-51.0)$ \\
\hline & SCC & 4 & $15.50(12.4-18.6)^{a}$ \\
\hline & EAC & 1 & - \\
\hline & Overall & 72 & $40.10(33.7-46.4)$ \\
\hline
\end{tabular}

SR, survival rate; Cl, confidence interval; $N$, number of included studies; ESCC, esophageal squamous cell carcinoma; SCC, esophageal small cell carcinoma; EAC, esophageal adenocarcinoma; Combined, esophageal cancer patients not classified by histology; N, number of included original studies. Between-group comparisons: ${ }^{a} P<0.05$ compared with ESCC group.

To address the strength and stability of the pooled results, we conducted sensitivity analysis by omitting one study at a time. The estimates of pooled survival were not significantly affected by any individual study that was included in our meta-analyses (see Figures S1-S4), indicating that our results had relative strength and stability.

\section{DISCUSSION}

Our study examined the hospital-based survival across China of patients with EC. Among the combined 79,777 patients from 331 hospital-based studies, the overall 5-years survival rate of EC was $40.1 \%$. Tendency analyses show that survival rates of EC have been increasing from 2000 to 2018. Subgroup analyses illustrate that men with EC have poorer survival compared to women.

In China, the regions with a high prevalence of EC are located in Fujian, Guangdong, Henan, Jiangsu, Shandong, Shanxi, Shaanxi, and Sichuan provinces (21-25). We found that EC survival rates in high prevalence areas were similar to those in other regions. It is notable that $90 \%$ of ECs in the Chinese population are ESCCs, which is inconsistent with other ethnicities. Our study found that survival of ESCC was significantly higher than SSC in China. As a minor histologic type of EC in China, EAC accounts for $<1 \%$ of EC (19). Among the hundreds of studies we included, only two reported an independent result on EAC. Due to the lack of such studies, the
TABLE 4 | The pooled survival and 95\% $\mathrm{Cl}$ of esophageal cancer at different sites.

\begin{tabular}{|c|c|c|c|}
\hline Categories & Sites & $N$ & SR (\%) \\
\hline \multirow[t]{5}{*}{ 1-year survival } & Hypopharynx and cervical & 6 & $76.5(68.7-84.4)$ \\
\hline & Upper thoracic & 15 & $79.3(73.7-84.8)$ \\
\hline & Mid thoracic & 19 & $77.6(72.1-83.0)$ \\
\hline & Lower thoracic & 15 & $73.3(66.2-80.3)$ \\
\hline & Overall & 55 & $77.1(73.9-80.3)$ \\
\hline \multirow[t]{5}{*}{ 2-years survival } & Hypopharynx and cervical & 2 & $69.4(45.0-93.7)$ \\
\hline & Upper thoracic & 3 & $49.1(25.9-72.3)$ \\
\hline & Mid thoracic & 8 & $44.5(35.4-53.6)$ \\
\hline & Lower thoracic & 5 & $34.9(6.4-63.5)$ \\
\hline & Overall & 18 & $44.7(36.2-53.1)$ \\
\hline \multirow[t]{5}{*}{ 3-years survival } & Hypopharynx and cervical & 9 & $40.4(30.2-50.6)$ \\
\hline & Upper thoracic & 16 & $48.1(42.3-54.0)$ \\
\hline & Mid thoracic & 20 & $44.5(35.2-53.8)$ \\
\hline & Lower thoracic & 16 & $46.8(39.7-53.9)$ \\
\hline & Overall & 61 & $45.6(41.0-50.1)$ \\
\hline \multirow[t]{5}{*}{ 5-years survival } & Hypopharynx and cervical & 3 & $10.7(0.1-21.4)$ \\
\hline & Upper thoracic & 11 & $32.6(24.8-40.4)^{\mathrm{a}}$ \\
\hline & Mid thoracic & 12 & $37.4(24.9-49.9)^{\mathrm{a}}$ \\
\hline & Lower thoracic & 12 & $39.6(31.0-48.1)^{\mathrm{a}}$ \\
\hline & Overall & 38 & $35.2(28.8-41.5)^{\mathrm{a}}$ \\
\hline
\end{tabular}

$S R$, survival rate; $\mathrm{Cl}$, confidence interval; $\mathrm{N}$, number of included original studies. Betweengroup comparisons: ${ }^{a} P<0.05$ compared with hypopharynx group.

pooled survival of EAC might not be representative. Moreover, TNM stage is also a crucial determinant for EC survival (26). Our findings showed a decreased trend of survival for patients with T1, T2, T3, and T4 stage EC. The 5-years survival rate of patients with stage T4 EC was nearly three times lower than that of patients with T1 EC. About half of ECs locate in the middle thoracic esophagus, followed by lower thoracic, higher thoracic, and hypopharyngeal parts of the esophagus (27). Our subgroup analyses showed lower survival rates among patients with hypopharynx and cervical EC, except for 2-years survival rates that were obtained from two published studies. These findings verify the relatively poorer prognosis of hypopharynx and cervical EC.

Because China has the highest burden of EC worldwide, our focus is to investigate the key measures of early detection and appropriate treatment for EC patients, as well as the strategies that can be taken to evaluate both the efficiency of preventive policies and the effectiveness of healthcare services across the country $(28,29)$. The national cancer registration system initiated in 2002 has been reinforced since 2015 and involved 416 population-based sites as of 2016 (30). Although this registry provides information on nationwide epidemics and burdens, it does not include all cancer patients in China. The comparability and validity of the information might vary between sites due to discrepancies in quality controls, as well as temporal heterogeneities. Moreover, follow-up is not easy to implement in the populations served by cancer registries. For instance, it has been reported that the follow-up rate was only between 65 and $71 \%$ at some sites (31). 

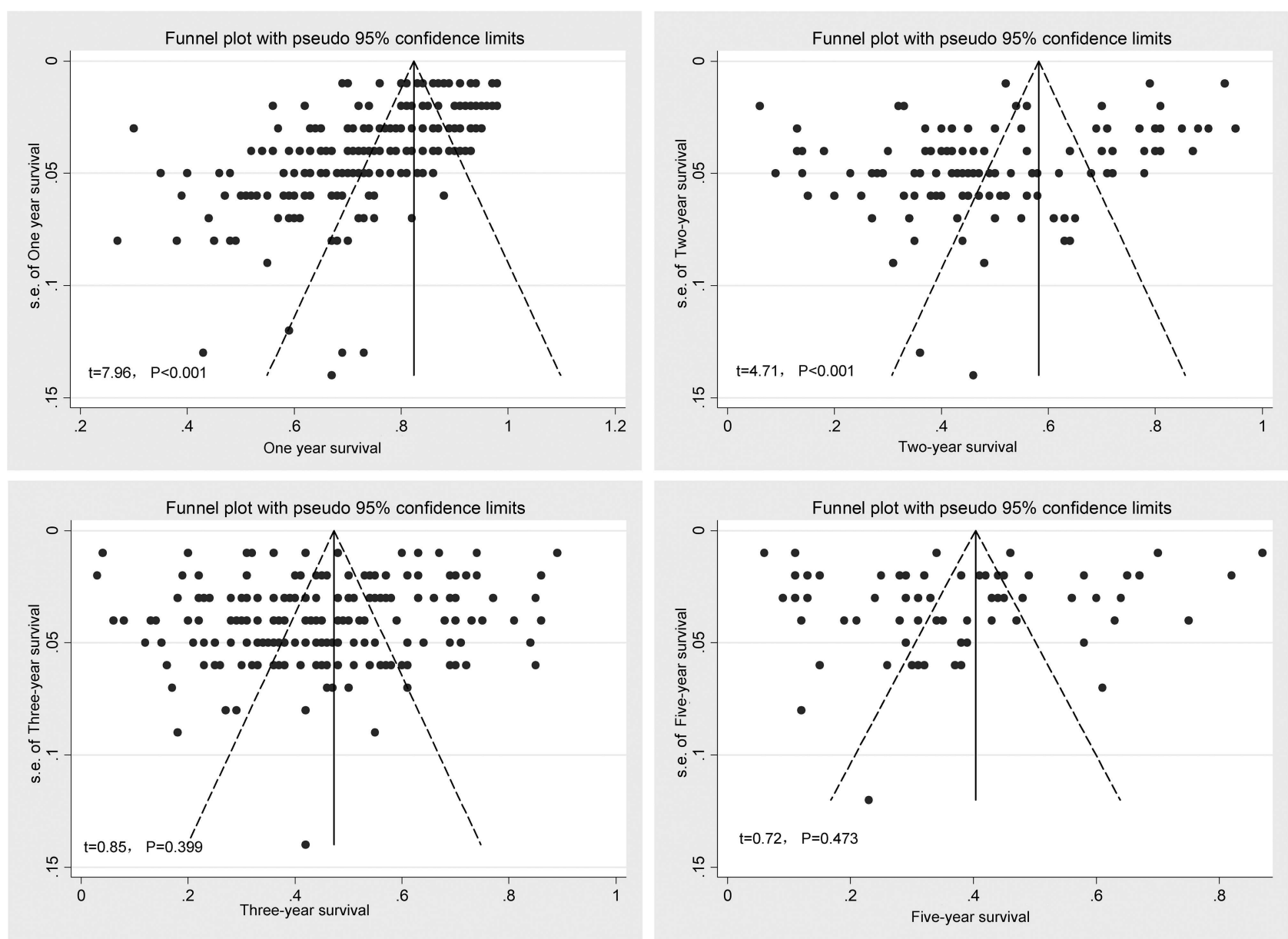

FIGURE 5 | Funnel plots of publication bias analyses.

Therefore, hospital-based studies are essential in order to predict the prognosis of patients with EC. We conducted this systematic review to combine the hospital-based survival data of patients with EC that were collected in accordance with clinical research criteria. We supposed that these results presented survival information from the viewpoint of clinical oncologists. Our findings verified an increased tendency toward EC survival from 2000 to 2018. The increase in EC survival has been attributed to improved access to primary health care, greater availability of diagnostic facilities, and improved effectiveness of clinical treatment. The health insurance system of China has been updated to cover all populations since 2003. Access to this insurance support has improved the survival of patients with EC across the country (11).

A large-scale study involving 63,506 patients with EC from 17 population-based cancer registries reported increased survival from 20.9 to $30.3 \%$ between 2003 and 2015 (12). However, we found a higher survival rate among the hospital-based population. This discrepancy is because population-based data are collected from non-inclusive study participants, while hospital-based data come from outpatients and inpatients who generally receive more effective therapy. Another population-based study estimated a decrease in survival from 19.1 to $18.1 \%$ in urban China (11), in which EC was the only cancer with a lower survival rate in urban compared to rural areas. Such results might be due to observation bias, which results from loss to follow-up of EC deaths in rural areas. In addition, it is known that in China, EC screening covered more rural areas than urban area prior to 2012. This might lead to the contrast fallacy between rural and urban populations. However, we did not analyze survival in urban or rural areas due to the lack of original information in our study. Age is also one of the important determinants closely related to the survival of EC. Unfortunately, we did not compare the survival between age classifications due to the lack of original data for age-specific groups.

In 2015, the third global surveillance of cancer survival (CONCORD) program reported survival data from 2000 to 2014, which included 734,428 adults with EC from 60 countries (32). During the 15-years period, 5-years age-standardized survival rates ranged from 10 to $30 \%$ in most countries, which was lower than what we observed. Data from a hospital-based cancer registry between 2002 and 2014 found that the 1-, 3-, 5-, and 10-years survival rates of EC were 55.88, 26.24, 19.62, and 
$12.14 \%$, respectively (15), which is also significantly lower than our results.

Limitations of our study are as follows: (1) Because the survival information is from independent studies, our findings are not representative of survival rates in China as a whole; (2) republication of survival data cannot be eliminated entirely; (3) most of the data in our study are from retrospective studies and might bias the real survival level of EC. In addition, significant publication bias was detected in both 1- and 2-years survival analyses. Positive publication bias indicates incomplete acquisition of original studies and might introduce potential fallacy to our observations.

In short, this systematic review and meta-analysis provides hospital-based survival data for EC in China. Although this study is subject to uncertain representability and obvious publication bias, it offers insight into the prognosis of EC based on inhospital studies.

\section{DATA AVAILABILITY}

All datasets generated for this study are included in the manuscript and/or the Supplementary Files.

\section{REFERENCES}

1. Jemal A, Bray F, Center MM, Ferlay J, Ward E, Forman D. Global cancer statistics. CA Cancer J Clin. (2011) 61:69-90. doi: 10.3322/caac.20107

2. Yu C, Tang H, Guo Y, Bian Z, Yang L, Chen Y, et al. Hot tea consumption and its interactions with alcohol and tobacco use on the risk for esophageal cancer: a population-based cohort study. Ann Intern Med. (2018) 168:489-97. doi: 10.7326/M17-2000

3. Wheeler JB, Reed CE. Epidemiology of esophageal cancer. Surg Clin North Am. (2012) 92:1077-87. doi: 10.1016/j.suc.2012.07.008

4. Tang WR, Chen ZJ, Lin K, Su M, Au WW. Development of esophageal cancer in Chaoshan region, China: association with environmental, genetic and cultural factors. Int J Hyg Environ Health. (2015) 218:12-8. doi: 10.1016/j.ijheh.2014.10.004

5. Chen W, Zheng R, Zeng H, Zhang S. The incidence and mortality of major cancers in China, 2012. Chin J Cancer. (2016) 35:73. doi: 10.1186/s40880-016-0137-8

6. Chen W, Zheng R, Baade PD, Zhang S, Zeng H, Bray F, et al. Cancer statistics in China, 2015. CA Cancer J Clin. (2016) 66:115-32. doi: 10.3322/caac.21338

7. Lin Y, Totsuka Y, Shan B, Wang C, Wei W, Qiao Y, et al. Esophageal cancer in high-risk areas of China: research progress and challenges. Ann Epidemiol. (2017) 27:215-21. doi: 10.1016/j.annepidem.2016.11.004

8. Cotton RG, Langer R, Leong T, Martinek J, Sewram V, Smithers M, et al. Coping with esophageal cancer approaches worldwide. Ann N Y Acad Sci. (2014) 1325:138-58. doi: 10.1111/nyas. 12522

9. Chen Q, Yu L, Hao C, Wang J, Liu S, Zhang M, et al. Effectiveness evaluation of organized screening for esophageal cancer: a case-control study in Linzhou city, China. Sci Rep. (2016) 6:35707. doi: 10.1038/srep35707

10. Wei WQ, Chen ZF, He YT, Feng H, Hou J, Lin DM, et al. Long-term follow-up of a community assignment, one-time endoscopic screening study of esophageal cancer in China. J Clin Oncol. (2015) 33:1951-7. doi: 10.1200/JCO.2014.58.0423

11. Zeng H, Zheng R, Guo Y, Zhang S, Zou X, Wang N, et al. Cancer survival in China, 2003-2005: a population-based study. Int J Cancer. (2015) 136:192130. doi: $10.1002 /$ ijc. 29227

12. Zeng $\mathrm{H}$, Chen $\mathrm{W}$, Zheng $\mathrm{R}$, Zhang S, Ji JS, Zou X, et al. Changing cancer survival in China during 2003-15: a pooled analysis of 17

\section{AUTHOR CONTRIBUTIONS}

YW and $\mathrm{HH}$ designed this study. ZM, XZ, and $\mathrm{HH}$ contributed to the literature search, review, and data extraction. MS and GD conduced statistical analyses. HH, $\mathrm{ZM}$, and $\mathrm{XZ}$ contributed to manuscript drafting. WW and YW contributed to manuscript modification. All authors have reviewed and approved the final version of this manuscript.

\section{FUNDING}

This study was supported by the Natural Science Foundation of Shandong Province, China (ZR2017MH100) and National Natural Science Foundation of China (81773527).

\section{SUPPLEMENTARY MATERIAL}

The Supplementary Material for this article can be found online at: https://www.frontiersin.org/articles/10.3389/fonc. 2019.00548/full\#supplementary-material

population-based cancer registries. Lancet Glob Health. (2018) 6:e555-67. doi: 10.1016/S2214-109X(18)30127-X

13. Chen JG, Zhu J, Zhang YH, Zhang YX, Yao DF, Chen YS, et al. Cancer survival in Qidong between 1972 and 2011: a population-based analysis. Mol Clin Oncol. (2017) 6:944-54. doi: 10.3892/mco.2017.1234

14. Brenner $\mathrm{H}$, Hakulinen T. Implications of incomplete registration of deaths on long-term survival estimates from population-based cancer registries. Int $J$ Cancer. (2009) 125:432-7. doi: 10.1002/ijc.24344

15. Chen JG, Chen HZ, Zhu J, Yang YL, Zhang YH, Huang PX, et al. Cancer survival in patients from a hospital-based cancer registry, China. J Cancer. (2018) 9:851-60. doi: 10.7150/jca.23039

16. Hou H, Zhao Y, Yu W, Dong H, Xue X, Ding J, et al. Association of obstructive sleep apnea with hypertension: a systematic review and meta-analysis. J Glob Health. (2018) 8:010405. doi: 10.7189/jogh.08.010405

17. Guo L, Yang F, Yin Y, Liu S, Li P, Zhang X, et al. Prevalence of human papillomavirus type-16 in head and neck cancer among the Chinese population: a meta-analysis. Front Oncol. (2018) 8:619. doi: 10.3389/fonc.2018.00619

18. Malhotra GK, Yanala U, Ravipati A, Follet M, Vijayakumar M, Are C. Global trends in esophageal cancer. Surg Oncol. (2017) 115:564-79. doi: $10.1002 /$ jso. 24592

19. Sun X, Zhao D, Liu Y, Liu Y, Yuan Z, Wang J, et al. The long-term spatial-temporal trends and burden of esophageal cancer in one high-risk area: a population-registered study in Feicheng, China. PLoS ONE. (2017) 12:e0173211. doi: 10.1371/journal.pone.0173211

20. Liu M, Zhang C, Cai H, Liu F, Liu Y, Li J, et al. The willingness to change risky health behaviors among Chinese rural residents: what we learned from a population-based esophageal cancer cohort study. PLoS ONE. (2016) 11:e0161999. doi: 10.1371/journal.pone.0161999

21. Sha $\mathrm{H}, \mathrm{Hu} \mathrm{D}, \mathrm{Wu} \mathrm{S}$, Peng F, Xu G, Fan G, et al. Baseline metabolic risk score and postsurgical esophageal cancer-specific mortality: the Fujian Prospective Investigation of Cancer (FIESTA) Study. J Cancer. (2018) 9:117381. doi: $10.7150 /$ jca. 23631

22. Liu S, Huang B, Huang H, Li X, Chen G, Zhang G, et al. Patrilineal background of esophageal cancer and gastric cardia cancer patients in a Chaoshan high-risk area in China. PLoS ONE. (2013) 8:e81670. doi: 10.1371/journal.pone.0081670 
23. Zhao JK, Wu M, Kim CH, Jin ZY, Zhou JY, Han RQ, et al. Jiangsu Four cancers study: a large case-control study of lung, liver, stomach, and esophageal cancers in Jiangsu Province, China. Eur J Cancer Prev. (2017) 26:357-64. doi: 10.1097/CEJ.0000000000000262

24. Han C, Qiao G, Hubbert NL, Li L, Sun C, Wang Y, et al. Serologic association between human Papillomavirus type 16 infection and esophageal cancer in Shaanxi Province, China. J Natl Cancer Inst. (1996) 88:1467-71. doi: 10.1093/jnci/88.20.1467

25. Liu X, Wang X, Lin S, Lao X, Zhao J, Song Q, et al. Dietary patterns and the risk of esophageal squamous cell carcinoma: a population-based case-control study in a rural population. Clin Nutr. (2017) 36:260-66. doi: 10.1016/j.clnu.2015.11.009

26. Shang QX, Yang YS, Xu LY, Li EM, Hu WP, Chen LQ. Prognostic significance and role in TNM stage of tumor deposits in esophageal cancer. J Thorac Dis. (2017) 9:4461-76. doi: 10.21037/jtd.2017. 10.60

27. Yang D, Mao YS, He J, Gao SG, Sun KL, Mu JW, et al. Long-term survival of the middle and lower thoracic esophageal cancer patients after surgical treatment through left or right thoracic approach. J Thorac Dis. (2018) 10:2648-55. doi: $10.21037 /$ jtd.2018.04.45

28. Hur C, Choi SE, Kong CY, Wang GQ, Xu H, Polydorides AD, et al. High-resolution microendoscopy for esophageal cancer screening in China: a cost-effectiveness analysis. World J Gastroenterol. (2015) 21:5513-23. doi: 10.3748/wjg.v21.i18.5513

29. Feng H, Song G, Yang J, Hao C, Wang M, Li B, et al. Cost-effectiveness analysis of esophageal cancer once-in-a-lifetime endoscopic screening in high-risk areas of rural China. Zhonghua Zhong Liu Za Zhi. (2015) 37:476-80. doi: 10.3760/cma.j.issn.0253-3766.2015.06.017

30. Wei WW, He J. Some thoughts on cancer registry in China: in the era of big data and informatization. Zhonghua Zhong Liu Za Zhi. (2019) 41:15-8. doi: 10.3760/cma.j.issn.0253-3766.2019.01.004

31. Investigation Group of Action Plan for Cancer Prevention and Control in China. Spot investigation report of action plan for cancer prevention and control in China. China Cancer. (2012) 21:641-3. doi: 10.11735/j.issn.1004-0242.2012.9.A001

32. Allemani C, Matsuda T, Di Carlo V, Harewood R, Matz M, Nikšić M, et al. Global surveillance of trends in cancer survival 2000-14 (CONCORD-3): analysis of individual records for 37513025 patients diagnosed with one of 18 cancers from 322 population-based registries in 71 countries. Lancet. (2018) 391:1023-75. doi: 10.1016/S0140-6736(17)33326-3

Conflict of Interest Statement: The authors declare that the research was conducted in the absence of any commercial or financial relationships that could be construed as a potential conflict of interest.

Copyright (c) 2019 Hou, Meng, Zhao, Ding, Sun, Wang and Wang. This is an openaccess article distributed under the terms of the Creative Commons Attribution License (CC BY). The use, distribution or reproduction in other forums is permitted, provided the original author(s) and the copyright owner(s) are credited and that the original publication in this journal is cited, in accordance with accepted academic practice. No use, distribution or reproduction is permitted which does not comply with these terms. 